\title{
Evaluación de la Calidad en Salud en la Medicina Familiar y en la Atención Primaria en Iberoamérica
}

\author{
Health Quality Assessment in Family Medicine and Primary Care in Ibero America \\ Avaliação da Qualidade em Saúde na Medicina de Familia e na Atenção Primária na Ibero-América
}

Maria Inez Padula Anderson. Departamento de Medicina Integral, Familiar e Comunitaria, Universidade do Estado do Rio de Janeiro (UERJ); Confederación Iberoamericana de Medicina Familiar. Rio de Janeiro, RJ, Brasil. inezpadula@yahoo.com.br (Autora correspondiente)

Macarena Moral. Universidad de Chile; Instituto de Administración de Salud, Chile. Chile. moralmacarena@ hotmail.com

Marcela Cuadrado Segura. Departamento de Medicina Familiar y Comunitaria, Universidad de la República del Uruguay; Sociedad Uruguaya de Medicina Familiar y Comunitaria; Grupo CIMF Medicina Familiar y Comunitaria en medio rural. Uruguay. marcelacua @gmail.com Thomas Meoño Martín. Escuela Medicina de la Universidad Autónoma de Centroamérica de Costa Rica; Asociación de Medicina Familiar y Comunitaria de Costa Rica; Caja Costarricense de Seguro Social. Costa Rica. drmeono@gmail.com Sergio Minué. Escuela Andaluza de Salud Pública de Granada. España. sergiominue21@gmail.com Ricardo Donato. Departamento de Medicina Integral, Familiar e Comunitaria, Universidade do Estado do Rio de Janeiro (UERJ). Brasil. ricardodonato2@yahoo.com.br

Lilia González. Escuela Nacional de Salud Publica. Cuba. lili@infomed.sld.cu

\section{Grupo de Trabajo}

Catalina Coral (Colombia), Christine Leyns (Bolivia), Felipe Moreno-Piedrahita Hernández (Ecuador), Jenny Magne (Bolivia), José de Almeida Castro Junior (Brasil), José Obando Romero (Costa Rica), Lisdamys Morera Gonzales (Perú); Pablo Aravia (Chile), Ajayrakeshvarma Chennareddy (India), Alejandra Paulo (Uruguay), Alexis Correa Taja (Nicaragua), Allison Acevedo (Colombia), Anahi Barrios (Uruguay), Anderson Mondragon (Colombia), Andrea Gamarra (Paraguay), Beatriz Salgado (Chile), Betsy Ballesteros Barragán (Colombia), Carmen Elena Cabezas Ecuador; Cecilia Llorach (Panamá), Daniel Capelli (Uruguay), Débora Teixeira (Brasil), Diana Yuruhan (Paraguay), Dora Bernal (Colombia), Dulce rivera (México), Edgar Leon (Ecuador), Enrique Falceto de Barros (Brasil), Esteban Cordero (Costa Rica), Gabriela Di Croce Argentina Gady Torres Ecuador, Garcia Vergara Figueroa (Brasil), Henry Solis Bolivia, Isabel Cristina Puello (Colombia), Jesús Martínez Ángeles (México), Jorge Brandão (Portugal), Juliana da Rosa Wendt (Brasil), Julio Braida (Uruguay), Karen Muñoz (Colombia), Maria Belen Giménez (Paraguay), Marina Almenas (Puerto Rico), Mauricio Alberto Rodríguez Escobar (Colombia), Mery Munive (Costa Rica), Noris Serrudo (Venezuela), Orlando Espinosa Bermúdez (Colombia), Oscar (México), Osvaldo García Torres (México), Patricia Taira Nakandoj (Brasil), Patricia Vargas (Chile), Philipp Oliveira (Brasil), Ricardo Anzures Carro (México), Ricardo Espitia (Colombia), Rodolfo Deusdará (Brasil), Verónica Casado (España), Victor Manuel Campos (El Salvador), Xenia Sancho (Costa Rica), Yolanda Flores (Ecuador)

Cómo citar: Anderson MIP, Moral M, Cuadrado MS, Meoño TM, Minué S, Donato R, et al. Evaluación de la Calidad en Salud en la Medicina Familiar y en la Atención Primaria en Iberoamérica. Rev Bras Med Fam Comunidade. 2016;11(Suppl 2):26-36. http://dx.doi.org/10.5712/rbmfc11(0)1389
Financiación:

ninguna declarada.

Aprobación ética:

La investigación fue considerada sin riesgo. Los autores declaran que los procedimientos seguidos se realizaron de acuerdo a las normas éticas de la Asociación Médica Mundial y la Declaración de Helsinki.

Conflicto de intereses: ninguna declarada.

Procedencia y revisión por pares: revisado por pares. Recibido el: 15/08/2016. Aceptado el: 15/09/2016. 


\section{Resumen}

El propósito de este estudio es contribuir al desarrollo del concepto y de los procesos relacionados a la evaluación de la Calidad en la Atención Primaria de Salud, bajo la perspectiva de Médicos de Familia y Comunidad (MFyC). Fue basado en la lectura de textos, discusión en grupo de trabajo y en una investigación con la aplicación de una encuesta semi estructurada a MFyC y otros profesionales provenientes de 19 países. Fueran obtenidas informaciones acerca de los servicios de AP y de las percepciones de sus procesos evaluativos, incluyendo los relacionados a la educación permanente, a la motivación y la sobrecarga de trabajo. Los resultados sugieren que evaluar de forma sistemática y adecuada la calidad de la AP en Iberoamérica aún es un proceso muy incipiente. Además, excepto por algunos pocos países, ni siquiera existe una APS universal con MFyCs en los equipos de salud. Por otro lado, si consideramos los principios y las practicas de la MFyC, parece haber una limitación de los instrumentos utilizados para evaluar la calidad en AP. Se concluye que para alcanzar una evaluación de calidad que pueda ser propulsora de una mejora continua de los servicios en la AP es necesario incluir indicadores relacionados a los conceptos y herramientas de la MFyC. Un concepto de Calidad en AP es propuesto y se indica las dimensiones a ser incluidas en los procesos evaluativos, considerando las competencias de la MFyC.

\section{Abstract}

The purpose of this study is to contribute to the development of the concept and the assessment of Quality in Primary Care, under the perspective of Family and Community Medicine (FCM). The study was based on texts reading, discussion in a working-group, and a survey with the application of a semi-structured questionnaire to FCM and other professionals from 19 countries. Information about PC services, as well as the perception of its evaluation processes, including those related to permanent education, motivation and work overload was obtained. The results suggest that the quality assessment of PC in lberoamerica is still a very incipient process. In addition, with the exception of a few countries, there is not even a universal PC with FCM in the health teams. Considering the principles and practices of the FCM, it seems that there is a limitation of the instruments commonly used to evaluate the quality in PC. It is concluded that to achieve a better quality assessment in order to conduct continuous improvements in the PC services, it is necessary to include indicators related to the concepts and tools of FCM. Considering the competences of the FCM, a quality concept in PC is proposed and dimensions to be included in the evaluation processes are indicated.

\section{Resumo}

O objetivo deste estudo é contribuir para o aperfeiçoamento do conceito e dos processos relacionados à avaliação da qualidade na Atenção Primária à Saúde (APS), sob a perspectiva de Médicos de Família e Comunidade (FCM). O estudo foi realizado com base na leitura de textos, discussão em grupo de trabalho e uma pesquisa com a aplicação de um questionário semi-estruturado a MFC e outros profissionais provenientes de 19 países. Foram obtidas informações sobre os serviços de APS e das percepções sobre seus processos de avaliação, incluindo os relacionados à educação permanente, motivação e sobrecarga de trabalho. Os resultados sugerem que avaliar adequadamente e sistematicamente a qualidade da APS na Ibero-América ainda é processo muito incipiente. Além disso, com exceção de alguns países, não existe sequer uma APS universal com MFCs nas equipes de saúde. Por outro lado, se considerarmos os princípios e práticas de MFC parece ser uma limitação dos instrumentos utilizados para avaliar a qualidade em APS, Conclui-se que para alcançar uma avaliação de qualidade que possa ser condutora de uma melhoria contínua dos serviços de APS é necessário incluir indicadores relacionados aos conceitos e ferramentas da MFC. Um conceito de Qualidade na APS é proposto e são indicadas dimensões para ser incluídas nos processos de avaliação, considerando-se as competências da MFC.
Palabras clave:

Calidad en Salud

Medicina Familiar

y Comunitaria

Atención Primaria

\section{Keywords: \\ Health quality \\ Family and Community \\ Medicine \\ Primary Care}

Palavras-chave:

Qualidade em Saúde

Medicina de Família

e Comunidade

Atenção Primária

\section{Introducción}

Desde Alma Ata - la Asamblea Mundial de Salud realizada en 1978 - se han experimentado avances importantes en la conformación e implementación de modelos de Atención Primaria de Salud (APS), especialmente en los países de economía más desarrollada, pero el mundo aún está lejos de alcanzar la meta establecida en aquella Conferencia. ${ }^{1}$ En los países de economía frágil, la baja implementación de una APS calificada es un problema relevante debido a una serie de barreras de índole conceptual, política, financiera y profesional. ${ }^{2} \mathrm{Ni}$ siquiera existe una norma nacional e internacional en relación con los conceptos y la integralidad de las acciones que deben existir para una APS de calidad. Estos países conviven diferentes modelos de APS que, en gran parte de las veces, están dirigidos al control de enfermedades puntuales, y son implementados a través de programas y paquetes verticales. ${ }^{3}$ 
En América Latina, la Medicina Familiar (MF), la especialidad de excelencia para el desarrollo de una APS de calidad, presenta distintas etapas de desarrollo. Excepto Cuba y México, no es necesario tener la especialidad para trabajar en la APS, al contrario de lo que ocurre en los países de economía más desarrollada, como Inglaterra, Canadá, Portugal o España. ${ }^{3}$ Pero, los tempos están cambiando una vez que están ocurriendo verdaderas reformas de los modelos de salud en algunos países de América Latina o, mismo en su interior, como es el caso de la ciudad Rio de Janeiro, en Brasil. ${ }^{4}$

En este contexto, y a medida que un modelo de salud basado en los principios de una Atención Primaria calificada sustituye un anterior, es necesario haber una reflexión por parte de los profesionales involucrados con su práctica, de manera que se revise sus objetivos. También, en este caso, los procesos evaluativos deben ser repensados para conseguir la excelencia en el ejercicio profesional y de la propia oferta de los nuevos servicios. Por lo tanto, se deben considerar de manera positivas las iniciativas de revisar los procesos evaluativos a nivel de la APS que sean planteadas de forma colaborativa por grupos de profesionales y sus asociaciones. La evaluación debe ser considerada, en este escenario, un instrumento clave para la mejora continua de la calidad de la Atención Primaria. ${ }^{5}$

Por otro lado, hay que evolucionar en el propio concepto de calidad en salud. Con el desarrollo de la humanidad, y con los avances en la biotecnología médica es necesario hacer un llamado para (re)humanizar la asistencia en salud y ofrecer servicios de mayor calidad, a partir de un enfoque holístico, incluyendo los recursos humanos, el compromiso, y la voluntad política que se necesitan para brindar una buena atención médica. Hay que pensar en el concepto de Calidad desde una perspectiva multidimensional, ${ }^{6}$ con con profesionales adecuados y con servicios de salud accesibles y equitativos. ${ }^{7}$ Además, incluir el concepto de Prevención Cuaternaria (evitar, reducir y paliar el perjuicio provocado por la intervención médica $)^{8}$ y también los pacientes, su satisfacción y expectativas con el servicio recibido como parte activa e importante dentro del proceso.

Es importante llamar la atención que la calidad posee una connotación histórico-cultural, es decir, es específica para una sociedad concreta. Son importantes los factores subjetivos o psicosociales de los individuos, los profesionales y la comunidad (creencias, valores, etc.). ${ }^{9} \mathrm{O}$ sea, la mejora de la calidad en AP debe constituir un objetivo permanente y debe adecuarse a los nuevos desafíos, tanto en función de la creciente complejidad de las necesidades de salud de la población, como por la transición epidemiológica y demográfica, y el actual contexto sociopolítico. ${ }^{10}$

Bárbara Starfield, ${ }^{11}$ estudió muchos sistemas de salud (en especial a partir de la década de 90 del siglo XX), y evidenció que las principales características que definen una APS de calidad tienen que ver con 7 atributos. Cuatro Atributos Esenciales: (1) atención de primer contacto, (2) longitudinalidad, (3) integralidad y (4) coordinación, y tres 3 derivados: (1) orientación familiar; (2) orientación comunitaria y (3) competencia cultural.

Considerando la hipótesis de que los procesos de evaluación de la calidad en APS están poco desarrollados en América Latina y en Iberoamérica, este estudio tiene por objetivo contribuir al desarrollo del concepto y de las prácticas relacionadas con la Calidad en Salud a partir de la visión de la Medicina Familiar y Comunitaria.

\section{Metodología}

Estudio de carácter exploratorio y abordaje cuali cuantitativa, desarrollado a partir de un Grupo de Trabajo, en el marco de las actividades pre, per y post la $6^{a}$ Cumbre Iberoamericana de Medicina Familiar, que tuvo lugar en San José de Costa Rica, en abril de 2016. Como proceso de trabajo el primer paso fue constituir el grupo con representantes de las Asociaciones de MFyC de Iberoamérica, ya en octubre de 2015. A continuación, se hizo un levantamiento bibliográfico con enfoque en los procesos evaluativos de la APS, valorando los que eran aplicados a nivel de país o región. En enero y febrero de 2016, a través de la colaboración del Grupo de Trabajo, se elaboró y se aplicó una encuesta semi-estructurada, a MFyC y otros profesionales interesados, oriundos de 19 países y respondida vía un cuestionario on line. En esta encuesta se buscó informaciones acerca de las características de los servicios prestados en la AP de los países, asimismo de las percepciones acerca de sus procesos evaluativos. Las informaciones obtenidas abarcaron: profesión, especialidad, país, conocimiento sobre si se hace evaluación de calidad en sus países, y las características de la evaluación: participación de los profesionales, periodicidad, adopción nacional o no; indicadores 
utilizados; planeamiento de las actividades en la unidad, tipos de servicios de salud prestados a la población, así como sobre la percepción de sobrecarga o de motivación para el trabajo. A continuación, el grupo se reunió para analizar y debatir los datos y, con bases en los resultados, hacer recomendaciones acerca del tema.

\section{Resultados}

\section{Estudios en calidad en la APS}

Fue hecha una pesquisa con las palabras de busca: calidad, atención primaria y medicina familiar, con el objetivo de encontrar algún estudio que evidenciase una perspectiva país o región, preferentemente en América Latina y/o Iberoamérica. Se encontró una revisión sistemática de la literatura, realizada en Brasil ${ }^{12}$ con el objetivo de identificar instrumentos nacionales e internacionales para la evaluación de la APS en los servicios de salud. En este artículo fueron identificados 3048 estudios, publicados entre 1979 e 2013. Los instrumentos validados en portugués, español o inglés y destacados en este artículo fueron: (1) WHO Primary Care Evaluation Tool (PCET); (2) ADHD Questionnaire for Primary Care Providers (AQ-PCP); (3) General Practice Assessment Questionnaire (GPAQ), PACOTAPS (Aplicativo para la APS); y (4) PCA Tools (Primary Care Assessment Tool), (5) EUROPEP (European Task Forceon Patient Evaluation of General Practice Care) y (6)P MAQ (Programa Nacional para el Mejoramiento del Acceso y Calidad de la Atención Primaria), utilizado en Brasil.

De entre estos, destacamos tres por presentar un carácter más amplio incluyendo, necesariamente, la organización y la práctica de la APS e involucrando los equipos de salud en el proceso evaluativo. También porque están siendo utilizados en una perspectiva internacional o regional o en países de América Latina como Brasil:

a) PCA Tool basado en el modelo de evaluación de la calidad de los servicios de salud propuesto por Donabedian ${ }^{13}$ - medición de estructura, proceso y resultados - y desarrollado por el equipo de Stafield en Johns Hopkins University Es constituido de 77 preguntas (ítems) en los siete atributos de la APS y permite, mediante respuestas de tipo Likert, obtener puntajes de 1-4 para cada atributo. Va dirigido a los profesionales de salud y la población atendida. No distingue si el profesional médico es Médico de Familia, o un médico generalista, o de cualquiera especialidad o si es un enfermero. También no abarca técnicas especificas de abordaje centrada en la persona, de abordaje familiar o comunitaria.

b) PMAQ-AB (2011), utilizado en Brasil para evaluar y promocionar el perfeccionamiento de la estrategia de Salud Familiar/APS, ${ }^{12}$ es un programa elaborado e implementado específicamente para el contexto de la APS. Abarca además, a los profesionales de salud que forman parte de los equipos, los usuarios y las diferentes instancias gestoras de todos los municipios. Fue creado por iniciativa gubernamental. Su principal objetivo es inducir la ampliación del acceso y la mejora de la calidad de la APS, garantizando un patrón comparable de calidad nacional, regional y local, para permitir mayor transparencia y efectividad de las acciones gubernamentales direccionadas a la APS. Se fundamenta, entre otros aspectos en los siguientes:

- La autoevaluación del equipo de salud: basados en las respuestas de los propios evaluados, incluso de los usuarios: estimula al colectivo a revisar el proceso de trabajo y a pensar en modos de cambiarlo, para superar problemas y alcanzar objetivos pactados por ese mismo colectivo.

- El monitoreo: registro de indicadores de atención y datos socio demográficos.

- La Educación Permanente: impulsada por las necesidades locales del equipo/comunidad, exigiendo de sus actores (trabajadores, gestores y usuarios) mayor capacidad de análisis, intervención y autonomía para el establecimiento de prácticas transformadoras.

- Además de la evaluación de los profesionales, es una Evaluación Externa, basada:

- En un proceso incremental, continuo y progresivo de mejora de los patrones e indicadores de acceso y calidad abarcando: gestión, proceso de trabajo y resultados alcanzados; 
- En la Transparencia en todas sus etapas, permitiendo el permanente seguimiento de sus acciones y resultados;

- En la Participación Voluntaria, tanto de los equipos de APS como de los gestores municipales; su éxito depende de la motivación y la pro-actividad de los actores involucrados.

c) EUROPEP - dedicado más específicamente a explorar la calidad en Atención Primaria desde la perspectiva del Usuario, - es un sistema de evaluación por los pacientes acerca de los servicios prestados por los médicos de familia. Fue desarrollado en los años noventa por EQUIP ${ }^{14}$ a través de la colaboración de representantes de diferentes países de Europa. Sus objetivos son mejorar la práctica, el rendimiento y la organización de la asistencia de los médicos de familia en la APS. El EUROPEP consta de 3 partes:

1. Indicadores clave (relación y comunicación, cuidado de la salud, información y apoyo, continuidad y cooperación, y organización de los servicios).

2. Áreas específicas de indicadores de satisfacción (consulta, agenda programada y accesibilidad, características del profesional, condiciones de los centros de salud y servicios prestados).

3. información sobre los usuarios (datos socioeconómicos y de salud y actitudes después de la experiencia de atención).

Tampoco distingue la especialidad del médico.

\section{Resultados de la encuesta}

Respondieron la encuesta: 81 profesionales oriundos de 19 países: Argentina, Bolivia, Brasil, Chile, Colombia, Costa Rica, Cuba, Ecuador, El Salvador, España, México, Nicaragua, Panamá, Paraguay, Perú, Portugal, Puerto Rico, Uruguay, Venezuela; dos procedían de otras regiones: India, Estados Unidos de América. Los respondientes fueron considerados en este estudio como informantes clave, ya que son representantes de las asociaciones de Medicina Familiar de Iberoamérica y/o fueron contactados por ellas. La distribución, en conformidad con la actividad laboral, fue la siguiente: Médicos de Familia y Comunidad 87,7\% (71 participantes); Residentes de MFyC: 9,.9\% (8); Gestor en Salud 2,.5\% (2).

En relación si se hacía evaluación sistemática, 41\% respondió que no. En el caso de las respuestas de que sí se hace, hay críticas por la calidad del instrumento, en cuanto a que no reflejaría adecuadamente si se brinda o no una atención de calidad (Figura 1).

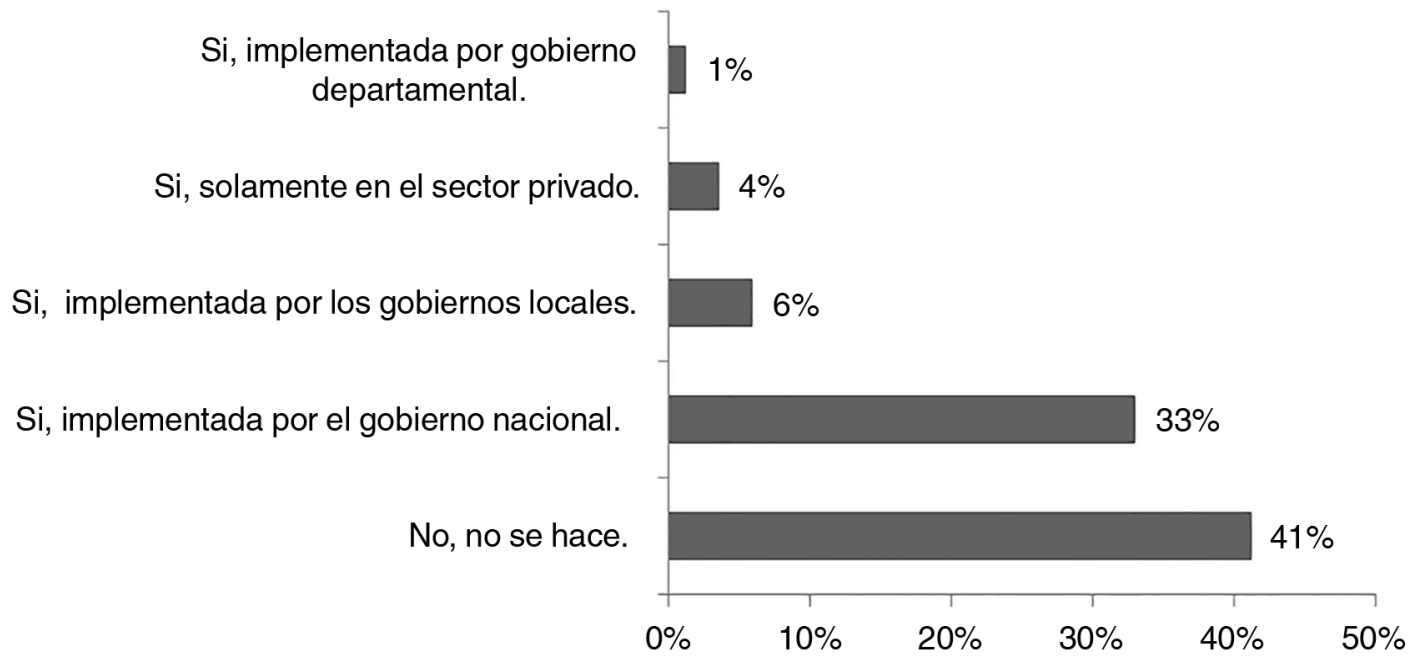

Figura 1. ¿En su país, se hace sistemáticamente la evaluación de la calidad de la Atención Primaria de Salud (APS)? Fuente: Encuesta CIMF, febrero de 2016 - Calidad en los Sistemas de Salud de Iberoamerica. 
Respecto de la periodicidad $41 \%$ informaron que se realizaba una vez al año y $39 \%$, semestral.

En cuanto a la percepción sobre la motivación y la carga de trabajo de los profesionales, el 91\% responde que no se evalúa de manera sistemática si los MFyC están motivados (Figura 2) y que $80 \%$ piensa que los MFCs de su país se sienten abrumados con la carga de trabajo (Figura 3 ).

$\square$ No, no se evalúa $\quad$ QSi periódicamente $\quad$ \&Otras respuestas

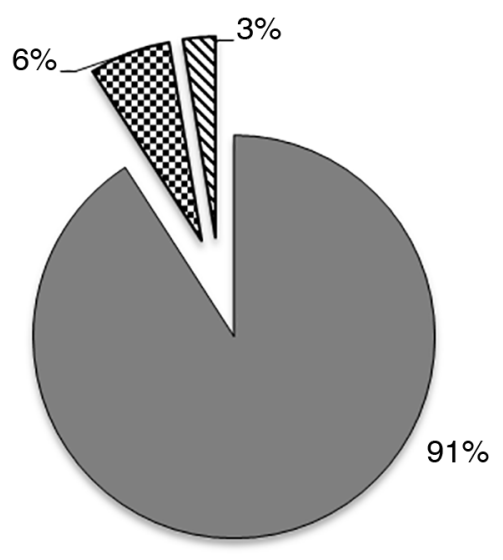

Figura 2. ¿En su país, se evalúa de manera sistemática si los MFyC están motivados para trabajar? (77 respuestas)

Fuente: Encuesta CIMF, febrero de 2016 - Calidad en los Sistemas de Salud de Iberoamerica.

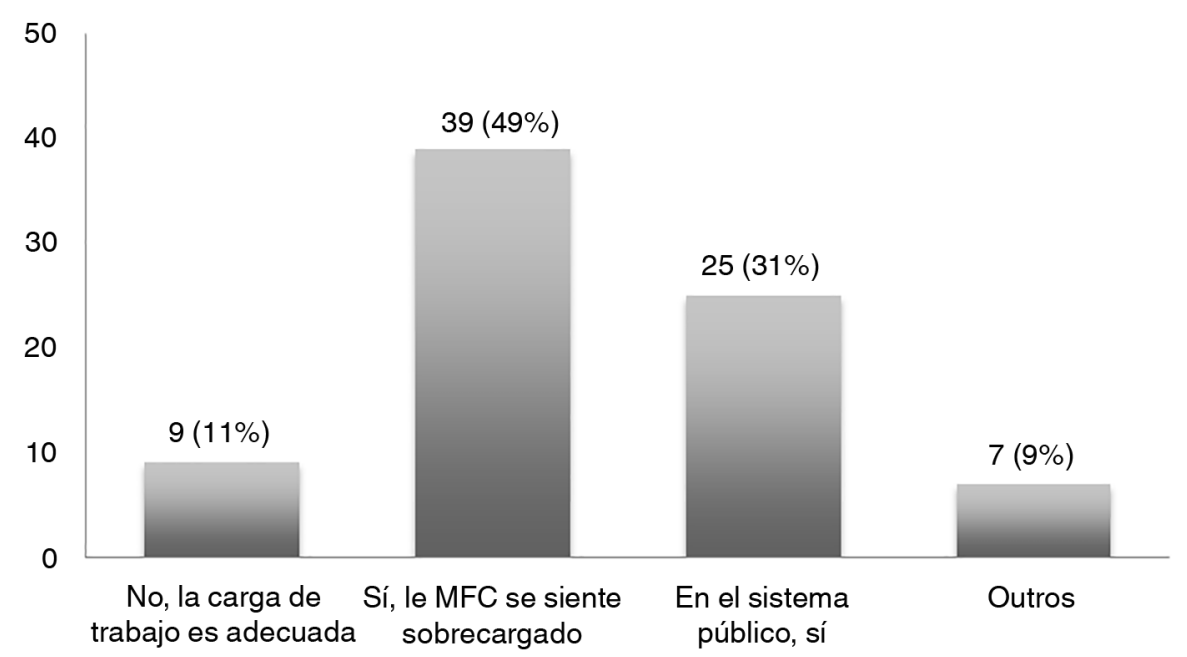

Figura 3. ¿En su país, el MFC se siente abrumado con la carga de trabajo?

Fuente: Encuesta CIMF, febrero de 2016 - Calidad en los Sistemas de Salud de Iberoamerica.

\section{Participación directa de los profesionales de salud en las unidades de APS en los procesos evaluativos de la calidad}

Cerca de $67 \%$ informa que no participa directamente y cerca del $29 \%$ informan que ellos mismos hacen la evaluación de los servicios. 


\section{Planeamiento de las acciones de salud y/o organización de los procesos de trabajo}

$35 \%$ informa que no se realizan reuniones periódicas con esta finalidad. De los que sí las hacen, la periodicidad es mensual en el $43 \%$, semanal en el $27 \%$ y semestral en el $22 \%$.

\section{El Desarrollo profesional continuo de los profesionales de salud se realiza de acuerdo con las necesidades de salud de la población local}

Cerca del 70\% respondieron que sí. En este tópico, algunos informaron que, muchas veces, los propios MFyCs no se interesan por las capacitaciones, y otros que los MFyC participan pero no el resto de los profesionales de la unidad.

\section{Indicadores de evaluación de la APS}

En relación a los indicadores de evaluación de la calidad en la APS, de los más utilizados son los cuantitativos (50\%); de prevalencia/incidencia de enfermedades (29\%). Solamente el $23 \%$ declara que su país utiliza indicadores de proceso para evaluar la APS (por ejemplo: tasa de disminución de Índice de Masa Corporal (IMC) en obesos; taza de interrupción de tabaquismo, entre otros).

De entre los atributos esenciales y derivados de la APS de acuerdo a Bárbara Starfield, los indicadores más utilizados en la evaluación de los participantes del estudio son los de acceso (34\%) y coordinación del cuidado (19\%). La competencia cultural, el abordaje comunitario, el abordaje familiar y la longitunalidad alcanzan menos de 15\%. (Figura 4).

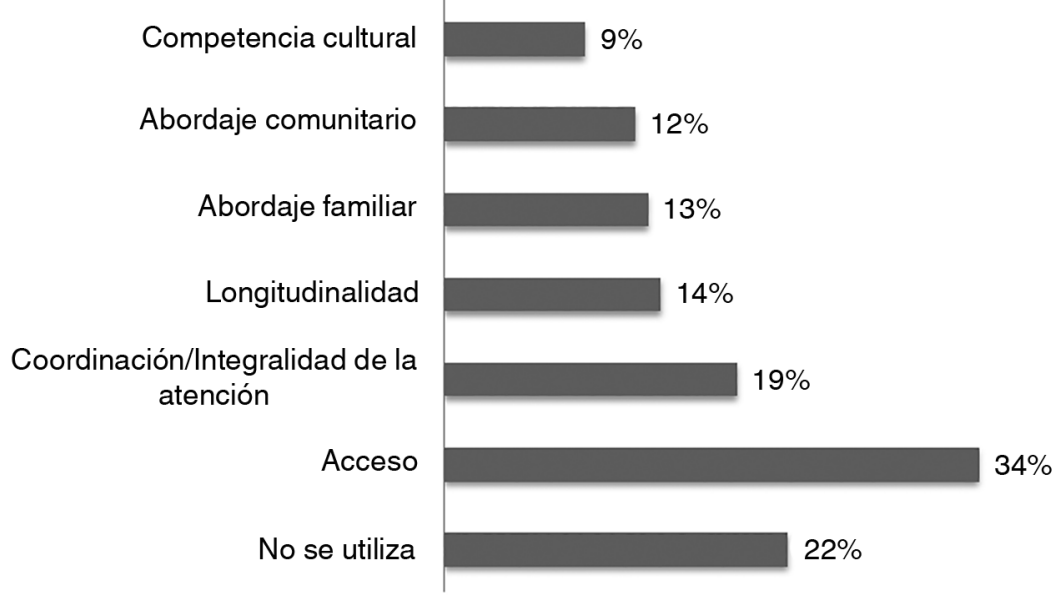

Figura 4. ¿Considerando los atributos esenciales y derivados de la APS (según Bárbara Starfield), indique los indicadores que se utilizan habitualmente en su país?

Fuente: Encuesta CIMF, febrero de 2016 - Calidad en los Sistemas de Salud de Iberoamerica.

\section{Servicios prestados en la APS}

Para la mayoría de los participantes (78\%), existe un paquete asistencial para la APS, pero para el $43 \%$ éste no es homogéneo para todo el país. Por otro lado, parece haber restricciones importantes para el acceso a exámenes y procedimientos desde las unidades de salud: el Electrocardiograma está disponible en el $48 \%$ de los casos, las pruebas de imagen mediante radiografía en el 38\%, la Ecografía en el 4\%, y otras Pruebas diagnósticas en el 46\%. Al mismo tiempo hay barreras, para el acceso a la medición de TSH (hormona tiro-estimulante), Hemoglobina glicosilada, o cintigrafía miocárdica. 


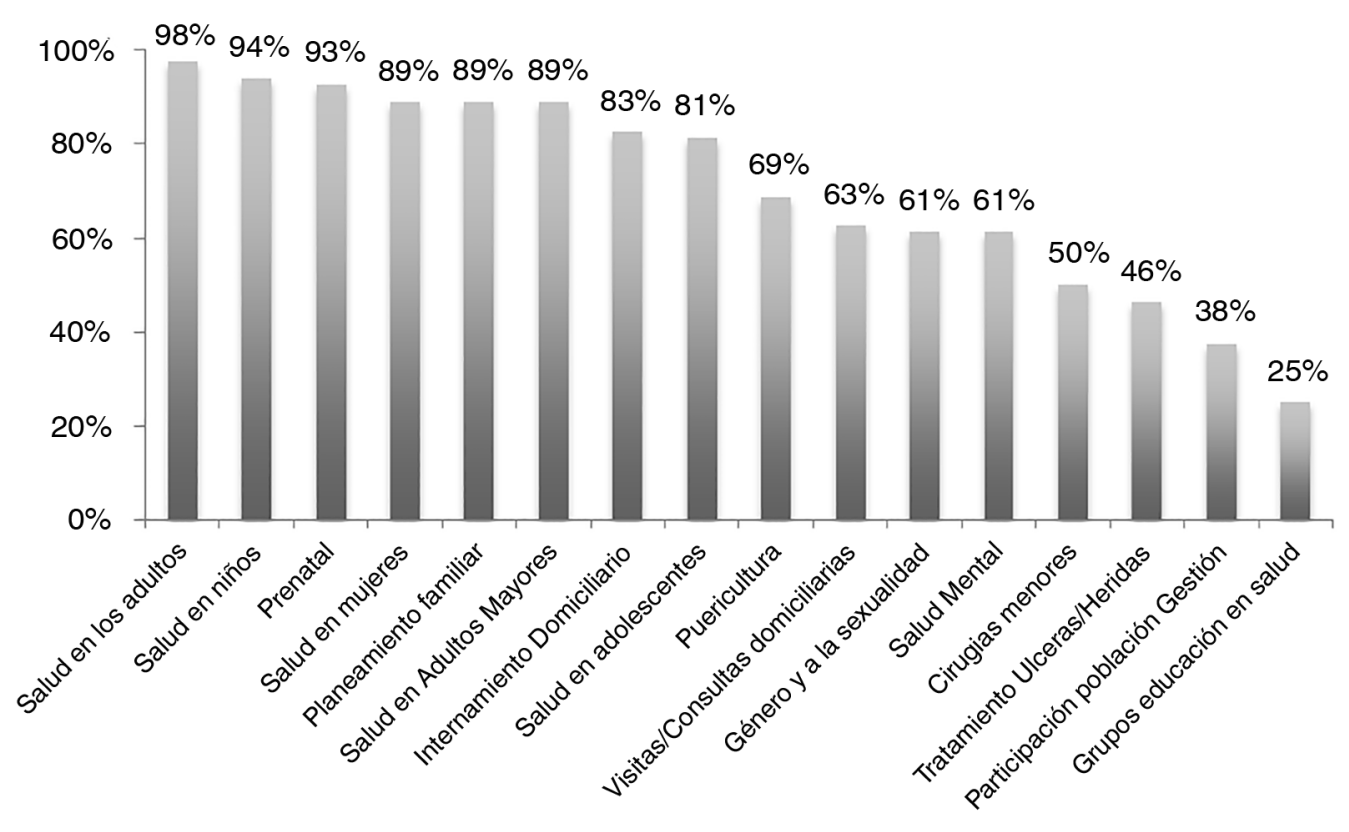

Figura 5. Servicios prestados en una unidad de Atención Primaria según los participantes.

Fuente: Encuesta CIMF, febrero de 2016 - Calidad en los Sistemas de Salud de Iberoamerica.

En relación a los tipos de servicios prestados en la APS, 69\% informan que la puericultura no se realiza en los servicios de APS. Por otro lado, las Visitas Domiciliarias, el Abordaje de temas como género y sexualidad, y la atención a la Salud mental se realizan en cerca del $60 \%$ de los casos. La participación social, y las actividades de grupos de educación en salud, solamente en el 38 y $25 \%$, respectivamente (Figura 5).

\section{Discusión}

Este estudio sufre de las limitaciones de uno estudio de carácter exploratorio. Por lo tanto tiene una perspectiva problematizadora y permite iniciar una reflexión más profundizada acerca de la práctica asistencial y de los procesos de evaluación en la APS a partir de la óptica de MFyCs en distintos países de América Latina. Levanta problemas potencialmente importantes en este proceso, como las posibles limitaciones de los instrumentos de evaluación existentes y la propia inexistencia de procesos sistemáticos de evaluación de la calidad.

Con base en el artículo de revisión de evaluación de calidad de la APS mencionado anteriormente, se podría decir que no se hace consideración específica acerca de la existencia o la necesidad de haber Médicos Familiares en los equipos como uno de los parámetros de calidad. Quizás porque la mayoría de estos estudios surgen en países donde la MF es una especialidad obligatoria y necesaria para trabajar en APS, por lo que no sería necesario explicitarlo. Pero ahí tenemos un problema potencial: cuando estos estudios se realizan en países donde la especialidad no es obligatoria, puede producirse el error de atribuir a la APS per se, con y sin MF, los buenos o malos resultados. También vale destacar que los estudios, en general, no abarcan instrumentos específicos utilizados en la práctica clínica cotidiana acerca del abordaje centrada en la persona, del abordaje familiar o comunitario. Las cuestiones que surgen en relación a estas dimensiones son pautadas por la impresión general del usuario o del profesional de salud acerca de su utilización o no. Puede ser que aquí también haga una percepción limitada por concepciones locales o más puntuales de lo que es la MFyC, de tal manera que la utilización de instrumentos específicos de su práctica estén colocados de manera general o basados en el sentido común. Agravante es además el hecho de que hay poca investigación sistemática sobre la organización o el desempeño de la APS y que no hay una visión crítica de los mecanismos institucionales para su monitoreo y evaluación. 
Relativo a los resultados de la encuesta, se destaca por ejemplo, el hecho de no haber investigación acerca de la motivación profesional o de la sobrecarga de trabajo de los MFyCs, ni tampoco acerca de la satisfacción de los usuarios.

Otro punto que merece destaque es que la mayoría informa que no hay participación directa de los profesionales de salud de las unidades de APS en los procesos evaluativos de la calidad, y que los indicadores de evaluación son, en su mayoría, cuantitativos, con una baja atención a los indicadores de proceso.

Llama la atención la limitación - o mismo inexistencia- de algunos servicios básicos en la APS, incluyendo la puericultura, las Visitas Domiciliarias, el abordaje en salud mental y de temas como genero y sexualidad. La actividades de participación social y de grupos de educación en salud, tampoco, por lo visto, son hechos en la medida esperada o necesaria.

Asimismo, parece haber poco interés en evaluar los atributos esenciales o derivados de la APS, como la coordinación, la longitudinalidad, la competencia cultural, el abordaje familiar y comunitario. Aún existen barreras de acceso a exámenes y si bien existan paquetes asistenciales para la APS, estos son insuficientes o no son homogéneos, incluso dentro de los propios países.

Como resultados positivos, podemos citar la existencia de planeamiento de las acciones de salud y/o de la organización del proceso de trabajo en la APS, en la mayoría de las respuestas. También, la existencia de un proceso de desarrollo profesional continuo para los profesionales de salud.

\section{Conclusión}

Implementar una APS universal en la América Latina con MFyCs en los equipos de salud aún es un reto. Evaluar la calidad de la APS en Iberoamérica es una tarea en desarrollo y falta mucho por hacer. Para que se logre hacer de una manera eficaz y atenta a los principios contemporáneos de calidad en salud y también de la propia MFyC, es fundamental involucrar a MFyCs en la elaboración, revisión, y propuestas de perfeccionamiento de los instrumentos existentes. Es necesario y estratégico incluir herramientas y aspectos específicos, no habitualmente abordados o incluidos de modo adecuado, cuando los parámetros de calidad son hechos solamente por otros profesionales o especialistas.

También es necesario involucrar a los MFyCs en las actividades prácticas relativas a los procesos evaluativos de la calidad de la APS, sea como evaluadores o como profesionales de salud en actuación. Es muy importante llamar la atención sobre la necesidad de explicitar la existencia, o no de MFyC en los equipos de salud, como uno de los factores esenciales para que una APS sea considerada de calidad.

Como contribución de este trabajo, a partir de las consideraciones y las propuestas contenidas en el, consideramos que:

- La CALIDAD de la Medicina Familiar y Comunitaria, como especialidad, y de la Atención Primaria como estrategia, deben evaluarse de manera interdependiente.

- Debe ser un proceso de mejora continua, basada en una evaluación cuali-cuantitativa, sistemática y dinámica.

- Debe servir para retroalimentar y perfeccionar las acciones de salud desarrolladas y dirigidas a la población asignada a una unidad de APS.

- Debe abarcar mecanismos auto-reguladores y de auto evaluación, involucrando a los profesionales de los equipos de salud, las personas, las familias y la comunidad asistida.

- Debe estar relacionada a un rol de principios y acciones a ser evaluadas de forma sistemática, permanente y dinámica, teniendo por objetivo el desarrollo y la mejoría continua de los Atributos Esenciales (Acceso/Puerta de Entrada; Coordinación del Cuidado, Integralidad, Longitudinalidad) y Derivados de la APS (Orientación Familiar; Orientación Comunitaria; Competencia Cultural), así como de la MFyC (Abordaje Biopsicosociocultural del proceso salud-enfermedad; Cuidado en Salud con enfoque en la persona, las familias y las comunidades, independiente del sexo, grupo de edad o estado de salud; Excelencia Clínica con elevada resolutividad para los problemas de salud más frecuentes presentados por una población adscrita). 
Debe abarcar, necesariamente indicadores multi-dimensionales relacionados a 10 grupos de elementos esenciales:

1. Los Atributos esenciales y derivados de la Atención Primaria.

2. Herramientas de la MFyC, con base en el paradigma biopsicosocial en una perspectiva sistémica, incluyendo el Abordaje Centrada en la Persona, Familiar y Comunitario, como por ejemplo, el uso de familiograma, de instrumentos de diagnostico comunitário, entre otros.

3. Cuidados en salud considerando los problemas y las necesidades de salud a lo largo de las vidas de las personas, familias y comunidad de una población asignada.

4. Equipo de salud mínimo para un determinado grupo poblacional/población asignada - necesariamente con médico de familia, enfermero y técnicos de salud.

5. Efectividad y equidad de los servicios de salud ofertados, considerando la ley de los cuidados inversos y la seguridad del paciente, y basado en los principios de la prevención cuaternaria.

6. La participación del equipo de salud y de la sociedad/comunidad organizada en los procesos diagnósticos de los problemas de salud y del planeamiento y gestión de de los servicios ofertados.

7. Desarrollo continuo de las competencias profesionales y personales de los miembros del equipo de salud, incluyendo su motivación y satisfacción para el trabajo.

8. Rol de la APS en el sistema de salud, considerando su integración, coordinación, y sistema de referencia y contra-referencia entre los diferentes niveles del sistema de salud, asimismo de otros actores inter-sectoriales.

9. Adecuación del proceso diagnóstico y terapéutico de acuerdo a las necesidades de salud, garantizando los recursos necesarios, incluidas las condiciones de estructura físico-funcional de las unidades de salud.

10. Experiencias positivas y de seguridad de las personas, familias y comunidades en relación al trato humano de los cuidados profesionales y de los servicios de salud realizados.

En base en lo que fue presentado y discutido en este grupo de trabajo, presentamos las siguientes directrices:

1. Proponer un modelo de evaluación de la calidad de la atención en APS y MFyC para Iberoamérica, a partir de los conceptos y características de calidad establecidos en este documento, considerando los instrumentos ya existentes y disponibles para uso, en especial, el PMAQ y el PCATOOLs, ambos ya traducidos para el español y portugués.

2. Organizar e implementar una investigación regional para que sea establecida una línea de base que permita evaluar los logros y acompañar la mejora continua de la calidad de la AP y de la MFyC en Iberoamérica.

3. Abogar por la implementación de un modelo de evaluación proprio de la AP y de la MFyC en los sistemas de salud de Iberoamérica y, quizá, a nivel mundial.

\section{Referencias}

1. Declaración de Alma Ata, disponible en: http://www.paho.org/spanish/dd/pin/alma-ata_declaracion.htm

2. Haq C, Ventres W, Hunt V, Mull D; Thompson, R; Rivo, M; Johnson, P. Donde no hay médico de familia: el desarrollo de la medicina familiar en el mundo/Wherethereis no family doctor: thedevelopment of familypracticearoundtheworld. Boletín de la Oficina Sanitaria Panamericana (OSP);120(1):44-58, ene. 1996.. http://hist.library.paho.org/Spanish/BOL/v120n1p44. pdf

3. Anderson MIP. Rojas Armadillo ML. Taureaux Díaz N Cuba Fuentes MS. Cobertura Universal en Salud, Atención Primaria y Medicina Familiar, Rev Bras Med Fam Comunidade. Rio de Janeiro, 2016 Ene-Mar; 12(Suppl 1):4-30 disponible en: https:// rbmfc.org.br/rbmfc/article/view/1276)

4. HOWE, Amanda; ANDERSON, Maria Inez Padula; RIBEIRO, José Mendes e PINTO, Luiz Felipe. 450th anniversary of the city of Rio de Janeiro: Primary Health Care Reform. Ciênc. saúde coletiva [online]. 2016, vol.21, n.5 [citado 2016-08-20], pp.13241326. Disponível em: http://www.scielo.br/scielo.php?pid=S1413-81232016000501324\&script=sci_arttext\&tIng=en 
5. J.R. Villalbí, M. Pasarín, I. Montaner, C. Cabezas, B. Starfield, et al. Evaluación de la atención primaria de salud, Aten Primaria 2003;31(6):382-5

6. García R.E. El concepto de calidad y su aplicación en Medicina, Rev. Med. Chile 2001; 129 (7).

7. Zurro AM; Gloria Jodar Solà GJ, Atención primaria de salud y atención familiar y comunitária -, Elsevier, 2011 - http://www. fmed.uba.ar/depto/medfam/bibliografia/Martin-Zurro.pdf

8. Gervas J, Gavilán E. JimenezL.Prevención cuaternaria: es posible (y deseable) una asistencia sanitaria menos dañina. AMF 2012;8(6):312-7http://amf-semfyc.com/web/downloader_articuloPDF.php?idart=994\&id=No_todo_es_clinica(14).pdf

9. Pérez CM, Ortiz-Reyes RM, Llantá-Abreu MC, Peña-Fortes M, Infante-Ochoa I. La evaluación de la satisfacción en salud: un reto a la calidad. Revista Cubana de Salud Pública. 2008 dic;34(4):0-0). http://bvs.sld.cu/revistas/spu/vol34_4_08/spu13408. htm

10. Ministerio de Saúde de Brasil.Secretaría de Atención de Salud. Departamento de Atención Primaria. Salud más próxima de usted. Acceso y calidad programa nacional de mejora del acceso y la calidad de la atención primaria (PMAQ): manual instructivo, 2012 http://bvsms.saude.gov.br/bvs/publicacoes/salud_mas_proxima_usted_acesso.pdf

11. Starfield B. Atenção Primária: equilíbrio entre necessidades de saúde, serviços e tecnologia. Brasil. Ministério da Saúde, 2002.

12. Fracolli LAG; Pereira MF, Zequini NFR, Santos Mariana Souza; Kelly CV, ACC Almeida Instrumentos de avaliação da Atenção Primária à Saúde: revisão de literatura e metassíntese. Ciênc. saúdecoletiva [Internet]. 2014 Dec [cited 2016 Apr 05]; 19(12): 4851-4860. Availablefrom: http://www.scielo.br/scielo.php?script=sci_arttext\&pid=S1413-81232014001204851\&Ing=en. DOI: http://dx.doi.org/10.1590/1413-812320141912.00572014

13. Donabedian A. Evaluación de la calidad de la atención médica. En: OPS/OMS, editores. Investigaciones sobre servicios de salud: una antología. Washington D.C.:OPS/OMS;1992.p.382-404. (Publicación Científica; 534).

14. European Society for Quality and Safety in Family Practice (http://equip.dudal.com/) 